\title{
Transfert et utilisation des résultats en milieu de travail : le cas de la recherche sur les éboueurs au Québec
}

Transfer and use of research results: the case of research into domestic waste collection in Quebec

Transferencia y utilización de los resultados en el ámbito del trabajo : el caso de una investigación sobre los basureros en el Quebec

\section{Madeleine Bourdouxhe et Laurent Gratton}

\section{(2) OpenEdition}

\section{Journals}

\section{Édition électronique}

URL : http://journals.openedition.org/pistes/3351

DOI : $10.4000 /$ pistes. 3351

ISSN : 1481-9384

Éditeur

Les Amis de PISTES

Édition imprimée

Date de publication : 1 mai 2003

Référence électronique

Madeleine Bourdouxhe et Laurent Gratton, «Transfert et utilisation des résultats en milieu de travail le cas de la recherche sur les éboueurs au Québec », Perspectives interdisciplinaires sur le travail et la santé [En ligne], 5-1 | 2003, mis en ligne le 01 mai 2003, consulté le 19 avril 2019. URL : http:// journals.openedition.org/pistes/3351 ; DOI : 10.4000/pistes.3351

Ce document a été généré automatiquement le 19 avril 2019.

\section{(c) (i) (9)}

Pistes est mis à disposition selon les termes de la licence Creative Commons Attribution - Pas d'Utilisation Commerciale - Pas de Modification 4.0 International. 


\title{
Transfert et utilisation des résultats en milieu de travail : le cas de la recherche sur les éboueurs au Québec
}

\author{
Transfer and use of research results: the case of research into domestic waste \\ collection in Quebec \\ Transferencia y utilización de los resultados en el ámbito del trabajo : el caso de \\ una investigación sobre los basureros en el Quebec
}

Madeleine Bourdouxhe et Laurent Gratton

\section{Objectifs}

1 Au moment de sa publication, qu'est-ce qui fait qu'une étude scientifique va être ou non utilisée par les personnes qu'elle concerne? Qu'est-ce donc qu'un transfert de connaissances « réussi »? Est-ce la dissémination de ces connaissances dans un public le plus large possible, ou est-ce plutôt l'utilisation effective de ces résultats par ceux qui en ont besoin? Quelles sont les conditions qui facilitent le processus de transfert? Existe-t-il une ou des formules gagnantes?

2 Le transfert des connaissances vers les milieux de travail ne va pas de soi et la seule évidence scientifique ne conduit pas forcément à des changements. Pour faire en sorte que les praticiens sur le terrain - travailleurs et employeurs - s'approprient les résultats de la recherche et les utilisent, certaines conditions sont essentielles.

3 L'objectif de cet article est d'apporter des éléments de réflexion autour de ce questionnement, essentiellement en illustrant le propos par une étude de cas : l'exemple du transfert des connaissances scientifiques issues des recherches sur la santé et la sécurité des éboueurs au Québec. Le texte fait l'inventaire rapide des conditions 
favorables au processus de transfert et présente brièvement quelques-unes des approches modélisées dans la littérature sur le sujet, en exposant de manière détaillée celle qui a été adoptée/adaptée par l'équipe de recherche. Enfin, comme il existe des limites à la diffusion des connaissances et à leur utilisation, il en sera discuté à la fin de l'article.

\section{Les étapes du processus de transfert}

4 La recension d'écrits scientifiques sur le sujet réalisée il y a quelques années par M. Roy et collègues mettait en évidence une série d'étapes du processus de transfert, à chacune desquelles les auteurs associaient ce qu'ils ont appelé des « conditions gagnantes » (Roy et coll., 1995).

\subsection{La création des connaissances}

La première condition essentielle à la réussite d'un transfert de résultats de recherche est d'y associer les principaux intéressés, les utilisateurs éventuels des résultats, dès le début $\mathrm{du}$ processus de création des connaissances. Ces dernières ont plus de chances d'être reçues et utilisées si les usagers potentiels sont associés à leur conception et si elles répondent bien à leurs besoins.

6 Dans le domaine de la recherche appliquée, le processus de création ne saurait se faire sans établir des relations interpersonnelles entre les chercheurs et les usagers. Une littérature abondante aborde cette notion sous différents vocables. Réjean Landry, directeur de la Chaire sur la dissémination et l'utilisation des résultats de la recherche de l'Université Laval, nomme ces relations interpersonnelles « le capital relationnel ». Il a démontré (Landry et coll., 2000) que le capital relationnel des chercheurs est le facteur le plus significatif dans le succès du transfert des résultats de la recherche sociale.

7 De son côté, en consultant la littérature sur le sujet, Mario Roy a lui aussi constaté (Roy et coll., op. cit.) que les communications interpersonnelles constituaient un véhicule majeur pour la dissémination de l'information scientifique. Les relations entre les chercheurs et les décideurs ont également été abordées par Jonathan Lomas dans le domaine de la recherche en santé ; selon lui, ces relations sont importantes pour améliorer la diffusion et l'utilisation des résultats (Lomas, 1997).

Dans ce contexte, les rôles d' « agent de liaison » ou de « portier » sont significatifs dans la mesure où ces acteurs jouent un rôle essentiel d'interface entre les chercheurs et les usagers. La difficulté de passer de la connaissance scientifique générée par les chercheurs à l'application pratique est directement proportionnelle à la largeur du fossé entre le chercheur et l'usager. En favorisant l'expression et la reconnaissance des besoins et des démarches de chacun, les liens interpersonnels, le capital relationnel, permettent de rétrécir ce fossé, et ce, dès le tout début du processus de production des connaissances, à l'étape même de la conception du protocole de recherche.

9 Le capital relationnel favorise non seulement la transmission des savoirs, mais il est avant tout un gage de la qualité même de la recherche, en particulier quand il s'agit d'analyser et de comprendre en profondeur les situations de travail réelles.

10 Bien entendu, le succès de la transmission des connaissances dépend du renforcement de ces liens interpersonnels tout au long du processus, très au-delà de l'étape initiale d'élaboration du protocole de recherche. 


\subsection{La transformation des connaissances}

11 Le simple fait de communiquer des résultats de recherche n'est pas suffisant pour qu'ils soient utilisés. Encore faut-il qu'ils soient adaptés aux utilisateurs et traduits dans un langage que ceux-ci comprennent. Bien plus qu'une simple question de vocabulaire commun entre chercheurs, relayeurs et utilisateurs, la compréhension implique l'intégration des éléments nouveaux produits par les uns au registre des connaissances que les autres se sont déjà construit au fil de leur expérience.

Quand la transformation de la connaissance est réalisée par des organismes qui ont l'habitude d'élaborer des solutions à des problèmes que connaissent bien les utilisateurs, alors le transfert ne peut qu'en être amélioré. C'est pour cette raison que le passage du langage proprement scientifique à une forme d'expression plus digeste pour les utilisateurs doit se faire avec l'aide de relayeurs. La participation des relayeurs au processus de transformation de la connaissance constitue une façon efficace de rendre les résultats de recherche utilisables.

\subsection{La diffusion des connaissances}

Lomas mentionne (idem) que les décideurs consultent peu les résultats de recherche dans leurs processus de décision, ou alors, quand ils le font, il faut que les recherches répondent immédiatement à leurs questions et besoins du moment.

«Il ne suffit pas qu'une recherche soit bonne pour qu'elle soit utilisée; elle doit répondre à un besoin immédiat et elle doit circuler à travers les réseaux appropriés pour être considérée en temps opportun ", disent de leur côté Roy et collègues (op. cit., 1995). Être à la bonne place, au bon moment, avec les bonnes personnes : voilà un autre critère de succès.

Lomas mentionne quatre sources de malentendus entre les chercheurs et les décideurs. La première est la tendance tant des chercheurs que des décideurs à traiter, de leur point de vue, soit la recherche ou la prise de décision, non pas comme un processus mais comme un produit. Une communication beaucoup plus systématique permettrait aux deux groupes de se comprendre. La deuxième source réfère à l'ignorance des chercheurs quant aux contextes politique et institutionnel de la prise de décision. Les troisième et quatrième malentendus sont les perceptions et les attentes des décideurs à l'égard des chercheurs et vice versa. Les décideurs voudraient que les chercheurs orientent toute leur recherche vers leurs intérêts immédiats tandis que les chercheurs ont tendance à traiter les décideurs comme un groupe homogène incapable de distinguer les besoins et les modes de diffusion propres à la recherche scientifique d'une part et à l'utilisation pratique d'autre part. Ceci dit, Lomas propose un ensemble de solutions et d'initiatives pour faciliter une meilleure communication et une compréhension mutuelle accrue entre les deux groupes. Ainsi, des colloques sont-ils organisés et des groupes de travail mis sur pied un peu partout au Canada pour en discuter (FRSQ, 1997; FCRSS, 1999; AWCBC/ ACATC, 2001). Ces activités ont pour effet de rapprocher les acteurs concernés et d'accroître les possibilités de diffusion et d'utilisation des connaissances. 


\subsection{La réception des connaissances}

Dans la hiérarchie des conditions favorables, la réception par les bénéficiaires potentiels des résultats est un autre élément clé pour une utilisation éventuelle. Toutes les conditions dites gagnantes concernant la création, la transformation et la diffusion des résultats sont vaines si les personnes auxquelles la connaissance est destinée ne sont pas réceptives à l'information qui leur est transmise. Pour être réceptif, il faut avoir confiance dans les agents de liaison qui diffusent cette connaissance, d'où l'importance de bien cibler les relayeurs, puisqu'ils sont des acteurs essentiels au transfert. Qu'est-ce qui fait l'importance des relayeurs? Quels sont leurs atouts? On en dénombre au moins cinq :

- leur mission d'intervention ;

- leur forte présence dans le milieu ;

- leur crédibilité ;

- leur pouvoir d'influence ;

- les véhicules de transfert dont ils disposent déjà dans leur milieu.

Dans le domaine de la santé et de la sécurité du travail au Québec, les relayeurs potentiels sont nombreux et œuvrent à divers paliers d'intervention auprès des décideurs, des gestionnaires, des travailleurs et des praticiens en prévention. Du côté patronal, les portiers et les relayeurs sont avant tout (ou en tout cas, ils devraient l'être au premier chef) les directeurs au niveau hiérarchique le plus élevé, les responsables des ressources humaines et de la formation, les médecins d'entreprise et le personnel médical, les directeurs de production (trop souvent oubliés alors qu'ils jouent pourtant un rôle capital en matière de prévention), les contremaîtres, les agents de sécurité. Du côté syndical, ce sont les délégués syndicaux, les représentants des comités de santé-sécurité, les responsables de la formation, voire les agents de grief et, bien sûr, les membres des exécutifs, de quelque niveau qu'ils soient. Il y a aussi tous ceux dont le métier même est la prévention : inspecteurs de la CSST, conseillers d'associations sectorielles paritaires (ASP) ou d'associations patronales en prévention. Mentionnons aussi les nombreux consultants privés : ergonomes, hygiénistes et préventionnistes et, finalement, tous ceux dont le métier consiste à véhiculer l'information: responsables internes des départements de communication ou ténors des médias externes, spécialisés ou non.

\subsection{L'utilisation des connaissances}

Les conditions d'utilisation des résultats de recherche se résument à la synthèse des éléments clés déjà évoqués pour les étapes précédentes du processus, soit l'engagement des relayeurs ciblés, l'utilisation de contacts interpersonnels, la planification concertée $\mathrm{du}$ processus de transfert et la transformation par les relayeurs en fonction de leur connaissance des récepteurs. Les éléments clés du transfert peuvent donc se résumer comme suit :

Encadré 1. Les éléments clés du transfert

La relation chercheur - utilisateur :

- Échanges continus entre les partenaires de recherche

- Association et engagement des utilisateurs dès le début du processus de création

Une stratégie de diffusion établie dès le départ : 
- Présence et engagement de relayeurs bien ciblés ayant la confiance des utilisateurs - Appropriation et transformation des résultats par les relayeurs - Accessibilité des résultats aux milieux de travail dans un langage s’intégrant aux compétences des utilisateurs

\section{Diverses approches dans le transfert de connaissances}

19 Il existe plusieurs approches pour décrire et modéliser le processus de transfert des connaissances scientifiques. Roy et coll. (op. cit., 1995) ainsi que Landry (1999) en mentionnent quelques-unes.

Une première distinction peut se faire en fonction de la source d'initiative du processus.

\subsection{L'approche " push »}

21 Cette approche met l'accent sur la disponibilité des résultats comme moteur aux transformations. L'avancement des connaissances inciterait automatiquement les décideurs et les utilisateurs à s'approprier les résultats de recherche. Plusieurs des conditions gagnantes mentionnées dans la section précédente n'étant pas remplies, il semble que cette approche concerne davantage la recherche fondamentale que la recherche appliquée.

\subsection{L'approche " pull »}

Ici, à l'inverse, ce sont les besoins qui motivent les décideurs et les utilisateurs à amorcer le processus. La difficulté de cette approche, caractéristique de la recherche appliquée, réside dans le fait que, en pratique, les usagers ont tendance à considérer les résultats de recherche comme des produits qui devraient être immédiatement disponibles et consommables. Ils insistent dès lors pour obtenir rapidement des réponses à leurs questions, peu soucieux de prendre en considération les contraintes épistémologiques et méthodologiques inhérentes au processus de recherche. La réciproque est également vraie, c'est-à-dire que les chercheurs ignorent souvent tout du processus décisionnel, d'où l'importance encore une fois des relations interpersonnelles entre les chercheurs, les décideurs, les relayeurs et les usagers.

Une autre façon de caractériser les types d'approches consiste à considérer le sens des échanges entre les acteurs du processus. Une approche n'est pas nécessairement meilleure que l'autre : tout dépend de la nature des projets, du type de résultats, de l'état de préparation du terrain récepteur et de la stratégie de transfert que l'organisation se donne.

\subsection{L'approche linéaire}

Le transfert de connaissances peut être unidirectionnel, c'est ce qu'on appelle l'approche linéaire (Dissanayake, 1986, cité dans Roy et coll., 1995). Elle suggère que les chercheurs transmettent leur savoir à des agents de liaison, des "portiers", des relayeurs, qui le diffusent auprès des usagers. Cette approche s'adapte bien à des résultats de recherche 
axés sur des bilans de connaissances, des innovations, des études documentaires et des recherches fondamentales. On parle ici plutôt de diffusion de connaissances puisque les connaissances produites par les chercheurs ont peu de liens (en terme de réciprocité, de synergie) avec les demandes des utilisateurs. L'écueil principal de cette approche est qu'elle ignore généralement les facteurs susceptibles d'influencer le transfert.

\subsection{L'approche d'interaction ou l'approche dite en spirale}

Le transfert de connaissances peut être aussi bidirectionnel. C'est l'approche d'interaction ou dite en spirale. Ici, l'importance des liens et des échanges entre les chercheurs et les utilisateurs est mise en évidence ; en fait, l'utilisation des résultats de recherche dépend, comme le nom l'indique, de l'interaction entre chercheurs et utilisateurs.

L'interaction est circulaire plutôt que linéaire comme dans l'approche précédente. Il y a réciprocité entre les acteurs. C'est l'intégration de la recherche à l'action et de l'action à la recherche. C'est la participation de tous les acteurs à toutes les étapes du processus de recherche, de la création à l'utilisation, condition essentielle à son appropriation (Bouchard et Gélinas, dans Gélinas, 1990). Notons d'ailleurs qu'en dépit de son ouverture, ce schéma pèche encore par excès d'étanchéité. En effet, le savoir théorique n'est pas l'apanage exclusif du chercheur, pas plus que le savoir d'expérience n'est celui du travailleur ou du décideur. À notre avis, au cours du processus, l'échange est tel que l'humain chercheur devient de plus en plus expert dans le «faire » et l'humain agissant de plus en plus habile dans le « voir » (figure 1).

Figure 1. L'approche en spirale

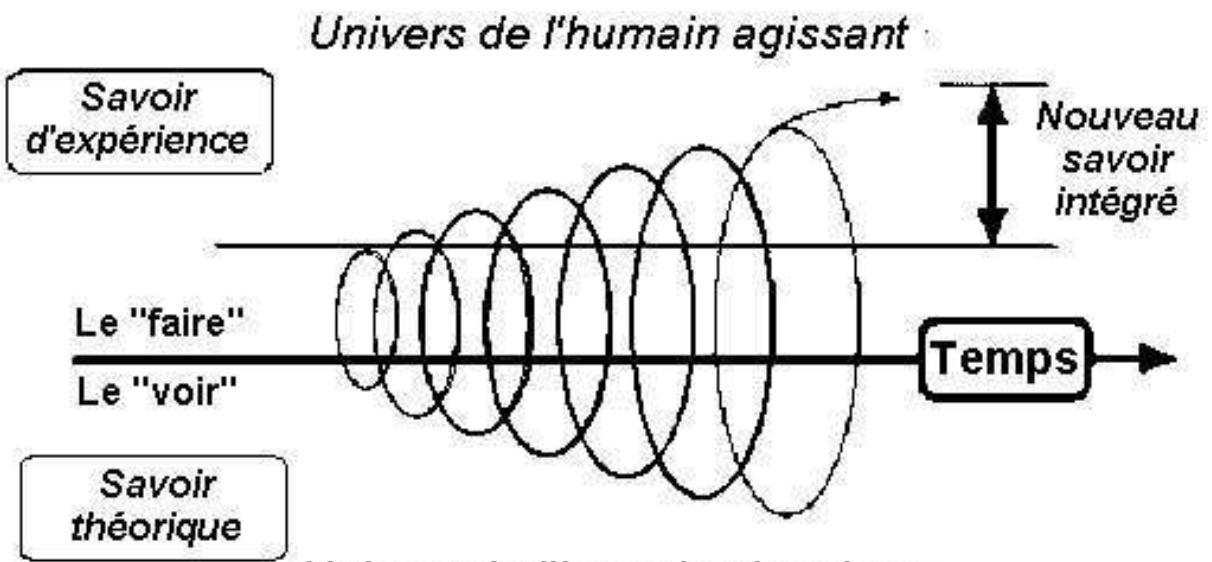

Univers de l'humain chercheur

Bouchard et Gélinas, 1990

27 L'approche en spirale se distingue par les caractéristiques suivantes :

- L'équipe de recherche s'adjoint un comité de suivi où la présence des relayeurs des résultats est sollicitée et où la demande initiale est réexaminée à la lumière de leur expérience.

- Dès le début de la recherche et tout au cours de celle-ci, les chercheurs s'attendent et sont disposés à modifier le protocole initial au fur et à mesure que l'expérience et les actions des utilisateurs potentiels seront mises à contribution. 
- Pour que le transfert des connaissances soit optimal et que les relayeurs s'approprient les résultats, les partenaires ont avantage à ce que la communication soit continue, avant, pendant et après la fin de la recherche. Et comme le mentionnent Huberman et GatherThurler (1991)

«Il est naïf de croire qu'un contact ponctuel avec les utilisateurs, en début ou en cours de recherche, créera une dynamique suffisante pour qu'ils s'investissent dans le transfert des résultats ".

- Le tableau 1 résume les caractéristiques des approches linéaire et en spirale.

Tableau 1. Principales caractéristiques de l'approche linéaire et de l'approche en spirale

\begin{tabular}{|c|c|}
\hline L'approche linéaire & L'approche en spirale \\
\hline $\begin{array}{l}\text { - La recherche est définie par le chercheur } \\
\text { - Le transfert de connaissances se fait par le } \\
\text { chercheur } \\
\text { - Le transfert de connaissances (la diffusion) se } \\
\text { fait surtout par des publications et des } \\
\text { présentations scientifiques ou professionnelles } \\
\text { spécialisées }\end{array}$ & $\begin{array}{l}\text { - Le projet s'associe un comité de suivi et la } \\
\text { présence de relayeurs } \\
\text { - Les actions et l'expérience des utilisateurs } \\
\text { peuvent entraîner des modifications au } \\
\text { protocole original du projet de recherche } \\
\text { - Il y a une communication continue entre } \\
\text { le ou les chercheurs et les utilisateurs } \\
\text { - Le transfert vise les utilisateurs et se fait } \\
\text { via des outils de diffusion qui les rejoignent }\end{array}$ \\
\hline
\end{tabular}

Les ergonomes sont d'ordinaire à l'aise avec l'approche en spirale, puisqu'ils travaillent aux frontières entre savoir et faire et qu'ils font des allers-retours incessants entre connaissance théorique et savoir pratique. Puisqu'on évoque l'ergonomie, il est intéressant de rappeler ici la contribution d'autres réflexions sur les transferts de connaissances, comme les travaux réalisés au laboratoire d'ergonomie du CNAM, notamment sur les rotativistes, sur le travail posté, ou lors de formations-actions auprès de militants syndicaux. Une collection intéressante de ces travaux est présentée dans un numéro spécial de la revue Éducation Permanente (Teiger et coll., 1995). Il y est dit que la formation dans les milieux de travail vise différents objectifs et revêt plusieurs formes. Elle constitue, pour l'ergonome comme pour le formateur, un moyen privilégié de transformer les situations de travail, et s'adresse à toutes les catégories d'acteurs en entreprise. Ce numéro pose des questions d'ordre scientifique mais aussi de pratiques sociales: les articles font référence à une trentaine d'expériences de formation à l'ergonomie dans les milieux de travail, réalisées dans plusieurs pays, qui font l'objet de fiches de synthèse. L'ensemble de ces expériences ont été présentées lors du XII ${ }^{\mathrm{e}}$ congrès de l'Association internationale d'ergonomie (IEA), qui se déroulait à Toronto (Canada) en 1994.

\section{Le type d'approche de transfert des connaissances utilisé par l'équipe de recherche}

29 L'approche privilégiée par les chercheurs et les relayeurs, dont cet article résume les travaux dans l'industrie de la collecte des déchets, a été bâtie à partir de la pratique, au fil des expériences, des réussites sur le terrain et des échanges avec les responsables du Service d'exploitation des résultats de la recherche de l'institution. Jusqu'ici elle n'avait 
guère été systématisée, encore moins a-t-elle donné lieu à des discussions d'ordre théorique, mais elle a fini par constituer peu à peu et de façon tacite une sorte de «bréviaire opérationnel » du transfert des résultats, puisque après tout, cela marchait. L'approche utilisée présente quatre caractéristiques.

1. Elle est d'abord mixte, puisqu'elle combine à la fois :

- l'approche push, en mettant les rapports de recherche à la portée de tout le monde, notamment par le site Web de l'institut de recherche concerné ;

- l'approche pull, dans la mesure où les études font le plus souvent suite à des demandes émanant directement des milieux de travail ;

- l'approche linéaire lorsqu'il s'agit de diffuser des recherches fondamentales ou des innovations technologiques préexistantes, des bilans de connaissances ou des études documentaires ;

- l'approche en spirale, puisqu'elle intègre simultanément les besoins, les préoccupations et les contraintes des usagers et ceux des chercheurs ainsi que les moyens de transfert appropriés.

1. Elle suscite et même demande une collaboration continue entre les acteurs.

2. Elle sollicite aussi la participation des partenaires à toutes les étapes du processus de création, de diffusion et d'utilisation de la connaissance, ce qui ne peut que favoriser son appropriation et son adoption.

3. Elle peut même aller jusqu'à inclure dans certains cas le soutien et la formation des relayeurs, comme l'indique l'exemple de la recherche sur les éboueurs dont la description suit.

\section{Illustration de l'approche : le cas du transfert des résultats de la recherche dans le secteur de la collecte des déchets domestiques}

Plusieurs auteurs ont insisté sur le besoin de rapprocher les systèmes de production de la connaissance des préoccupations des usagers. Si l'on veut que le processus de transfert soit effectif et le plus abouti possible, on a avantage à mettre l'accent sur les réseaux d'acteurs impliqués dans le processus de création, de diffusion et d'utilisation plutôt que sur les mécanismes de transfert en soi. L'efficacité de cette stratégie est illustrée par deux études qui ont été effectuées dans le secteur de la collecte des déchets domestiques (étude des accidents : Bourdouxhe et coll., 1992 ; étude des risques reliés aux bioaérosols : Lavoie, 2000). Nous montrerons comment les conditions exposées précédemment ont été mises en place pour que le transfert réussisse, en faisant ressortir toutefois, dans la discussion, les limites à ce transfert.

\subsection{La création de la connaissance : la recherche proprement dite}

\subsubsection{Le contexte de la recherche}

31 En 1990, il n'existait pas de données statistiques ni d'étude scientifique sur la collecte des ordures ménagères, alors que des risques nombreux et coûteux semblaient reliés à ce travail. Les associations sectorielles paritaires en santé et sécurité des secteurs Transport - entreposage et Affaires municipales (ASTE et APSAM) du Québec ont alors demandé que cette industrie fasse l'objet d'une étude. En 2000, c'était au tour de la Commission de la santé et de la sécurité du travail (CSST) de demander des bases scientifiques pour amorcer 
un Programme d'Intervention Intégré (PII) sur les risques biologiques chez les éboueurs. La « coïncidence » n'a probablement rien de fortuit, cette deuxième demande étant sans doute liée à la réussite du transfert de connaissances de la première.

La population visée regroupe, outre les 250 travailleurs des 5 municipalités du Québec qui collectent leurs déchets domestiques sous juridiction municipale directe, plus de 2500 éboueurs et chauffeurs qui travaillent pour les 350 entreprises privées sous-traitantes.

\subsubsection{La transformation de la demande}

Il arrive souvent que la demande initiale doive être révisée parce qu'elle s'avère inadéquate par rapport aux besoins réels des clients. À cet égard, le cas de l'étude des accidents est exemplaire. Au tout début, il s'agissait d'évaluer la pertinence d'un projet de recherche soumis par un consultant à l'organisme subventionnaire. Il aurait consisté à concevoir un pare-éclats destiné à protéger les éboueurs des projections de substances ou objets dangereux lors de la compaction du contenu de la trémie du camion à chargement arrière. Une étude préliminaire des dossiers d'accidents de trois entreprises a très vite révélé que les accidents par projection étaient quasiment inexistants, alors que de nombreux accidents survenaient dans des circonstances variées impossibles à cerner faute de connaître le travail réel des éboueurs et ses risques. Dès lors, c'est ensemble que chercheurs et clients ont reformulé la demande, ce qui constitue en fait la première boucle de l'approche en spirale.

\subsubsection{Les objectifs des deux études}

Dans la première étude (1992), le but était de dénombrer et décrire les accidents, de comprendre comment ils surviennent en analysant le travail des éboueurs et en étudiant l'organisation et la gestion des parcours de collecte, et finalement de proposer des solutions orientées vers la prévention à la source. L'objectif de la deuxième étude (2000) était de caractériser les expositions des éboueurs aux bioaérosols en tenant compte des facteurs qui influencent les conditions de travail, et de proposer ensuite des solutions pour contrôler les expositions.

\subsubsection{La méthodologie, les résultats, les recommandations}

Le tableau suivant résume les méthodologies employées dans chacune des études, les principaux résultats et les principales recommandations qui en découlent (tableau 2).

Tableau 3. Les deux études sur les éboueurs : méthodologies, résultats, recommandations

\begin{tabular}{|l|l|l|}
\hline & $\begin{array}{l}\text { Accidents, tâches, organisation du travail } \\
(1992)\end{array}$ & Bioaérosols (2000) \\
\hline
\end{tabular}




\begin{tabular}{|c|c|c|}
\hline $\begin{array}{l}\text { Méthodes : Études } \\
\text { de terrain }\end{array}$ & $\begin{array}{l}300 \text { heures d'observation ( } 29 \text { tournées, } 2800 \\
\mathrm{~km}, 21 \text { heures de vidéo, } 7 \text { entreprises } \\
\text { privées et } 2 \text { municipalités, } 3 \text { métiers, } 3 \text { types } \\
\text { de territoires, } 3 \text { types de collectes, } 2 \text { types } \\
\text { de véhicules, toutes saisons) } \\
\text { Statistiques de } 755 \text { accidents } \\
\text { Évaluation ergonomique de } 32 \text { camions } \\
\text { Entrevues avec } 58 \text { travailleurs et } 9 \\
\text { gestionnaires (risques du métier, gestion de } \\
\text { la main-d'œuvre, des parcours, des } \\
\text { véhicules) } \\
\text { Mesure des expositions personnelles aux } \\
\text { bioaérosols (bactéries totales, endotoxines, } \\
\text { moisissures) dans } 7 \text { types de collectes l'été } \\
\text { (=pires conditions) } \\
\text { Évaluation des effets du nettoyage d'un } \\
\text { camion sur l'exposition }\end{array}$ & 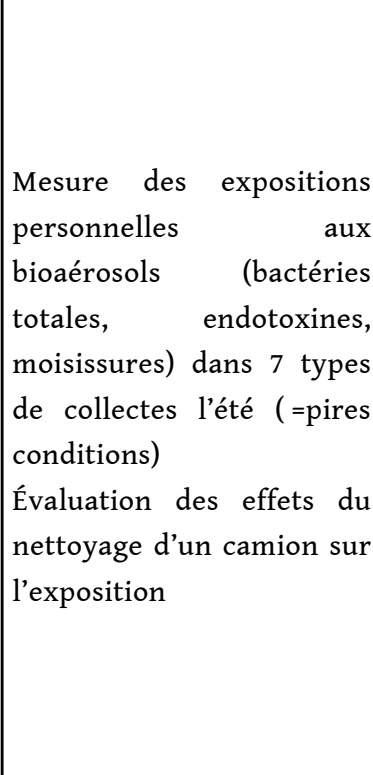 \\
\hline Résultats & 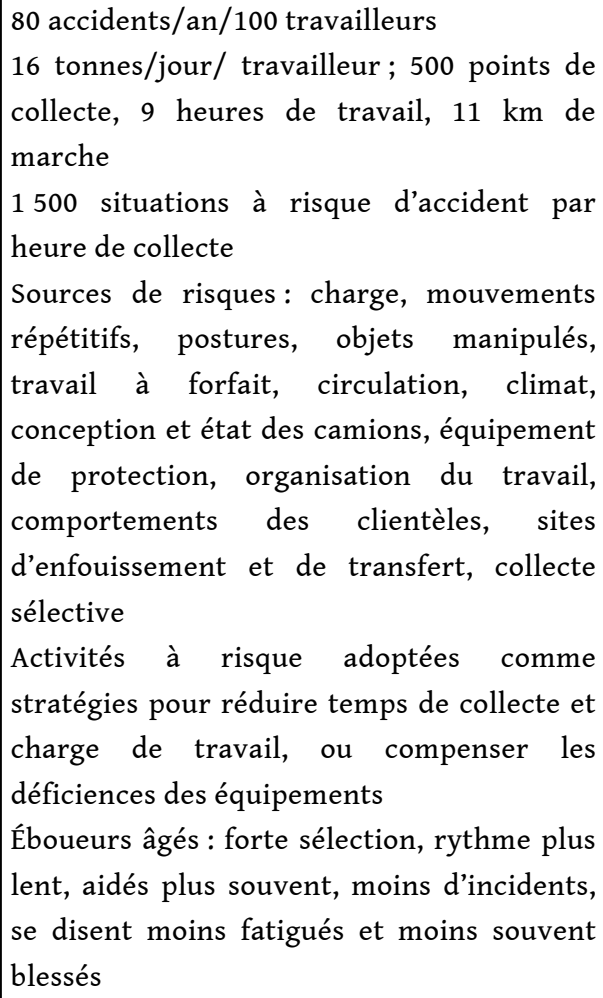 & $\begin{array}{l}\text { Expositions supérieures } \\
\text { aux valeurs guides pour les } \\
\text { bactéries et les } \\
\text { endotoxines dans la } \\
\text { collecte du compost } \\
\text { Six fois sur sept, la } \\
\text { concentration moyenne en } \\
\text { moisissures dépasse les } \\
\text { niveaux de base } \\
\text { Le nettoyage du camion } \\
\text { n'améliore pas la qualité } \\
\text { de l'air et un camion sale } \\
\text { ne constitue pas une } \\
\text { source majeure de } \\
\text { bioaérosols }\end{array}$ \\
\hline Recommandations & $\begin{array}{l}60 \text { recommandations dans } 10 \text { domaines, } \\
\text { dont embauche-formation, organisation des } \\
\text { parcours et charge de travail, aménagement } \\
\text { des camions, gestion publique, contrats de } \\
\text { collecte, stabilité de la main-d'œuvre, } \\
\text { gestion de la santé-sécurité }\end{array}$ & $\begin{array}{l}\text { Modifier le type de } \\
\text { collecte, ou favoriser la } \\
\text { protection respiratoire et } \\
\text { la possibilité (le temps) de } \\
\text { prendre des mesures } \\
\text { d'hygiène personnelle }\end{array}$ \\
\hline
\end{tabular}




\subsection{La stratégie de transfert} gagnantes » (Roy et coll., 1995), la réussite du transfert de connaissances s'appuie sur quatre postulats : la recherche répond à des exigences de qualité, les résultats seront relayés par des partenaires associés dès le départ, ils seront diffusés dans les milieux de travail non seulement par les véhicules de communication classiques, mais également transformés en outils et moyens accessibles à une variété de clientèles sur le terrain. Voici comment ces préceptes ont été illustrés dans la recherche sur les éboueurs.

\subsubsection{L'évaluation et la diffusion dans le milieu scientifique}

Les utilisateurs sont exigeants, à juste titre. Ils décident de changer leurs pratiques seulement si les résultats de recherche qui leur sont proposés sont crédibles et que leur qualité scientifique a été éprouvée. Les études dont il est question ici ont été évaluées à plusieurs étapes, de l'examen de la pertinence et de la priorité des projets par le conseil scientifique de l'institution accordant les fonds de recherche, à l'évaluation de la qualité scientifique, tant des protocoles que des rapports, par des comités de pairs externes. Outre les monographies publiées par l'institut de recherche concerné, les études sur la collecte des déchets et sur les bioaérosols ont fait l'objet d'articles dans des revues avec comités de pairs et de communications dans des congrès scientifiques. Comme il arrive souvent, la confirmation de la valeur scientifique de ces études dans les milieux de recherche par des publications s'est produite après que les résultats aient été transmis aux principaux intéressés. Comment répondre aux attentes du milieu et aux besoins d'action tout en conservant l'intégrité scientifique? Le sérieux (certains parlent de lourdeur) du processus d'évaluation institutionnel répond à ces préoccupations; une façon complémentaire ou alternative de résoudre ce problème serait d'adjoindre des scientifiques au comité technique, ou encore de mettre sur pied un comité scientifique chargé de conseiller les chercheurs sur ces aspects tout au long du projet ou du programme de recherche.

\subsubsection{Le choix des relayeurs et des partenaires dès le début de la recherche}

Le choix des relayeurs est un facteur d'autant plus important que le relais joue dans les deux sens, pour le bénéfice des utilisateurs mais aussi celui des chercheurs. Plus ceux-ci sont en prise directe avec le terrain dès la conception du protocole de recherche, plus ils seront capables d'ajuster leur méthodologie aux réalités du milieu et de poser les «bonnes» questions; plus la confiance réciproque est élevée, plus l'information recueillie par les chercheurs aura des chances d'être valide et fiable. Ceci est particulièrement important dans les études qui questionnent l'organisation du travail. Indépendamment de l'évaluation scientifique par les pairs, le comité de suivi sert aussi à valider les résultats (le milieu se reconnait-il dans le portrait ainsi dressé ?) et les recommandations (sont-elles réalistes ?).

Comme le recommande l'approche en spirale, dès le début des travaux, un comité de suivi paritaire s'est donc constitué pour piloter la recherche, la publiciser, ouvrir tous les accès au terrain, relayer l'information du terrain vers les chercheurs et réciproquement et préparer la stratégie de diffusion des résultats. Aux associations sectorielles Transport -

Perspectives interdisciplinaires sur le travail et la santé, 5-1 | 2003 
entreposage et Affaires municipales se sont joints des représentants de municipalités et d'entreprises privées, d'associations syndicales (Teamsters et Fédération des travailleurs et travailleuses du Québec) et patronale (Association des entrepreneurs de services en environnement), la Commission de la santé et de la sécurité du travail (CSST), les régies régionales en santé et services sociaux (RRSSS) et les centres locaux de services communautaires (CLSC, Équipes de santé au travail).

\subsubsection{La diffusion par les chercheurs dans les milieux de travail et dans le grand public par les moyens traditionnels}

L'engagement des chercheurs est indispensable à la diffusion des résultats. Leurs interventions ont donc été nombreuses, des présentations aux colloques organisés par les milieux de travail (Association des entreprises de services en environnement du Québec, Réseau environnement, municipalités, syndicats) jusqu'aux publications vulgarisées (10 articles dans des revues spécialisées en environnement et en santé et sécurité), en passant par les séminaires professionnels donnés aux ergonomes (Les journées de la pratique de l'ACE), les cours de formation pour les futurs ergonomes (École Polytechnique, Université de Montréal), les émissions de radio et de télévision et les communiqués et conférences de presse visant l'ensemble des médias québécois.

\subsubsection{La transformation des résultats de recherche en langage accessible et en outils utilisables par les milieux de travail}

41 Tous les auteurs s'entendent pour dire que les résultats de recherche deviennent pleinement accessibles aux principaux utilisateurs seulement lorsqu'ils sont traités, réorganisés et adaptés avec leur participation. Dans notre exemple, l'appropriation et la transformation des résultats par les relayeurs est assez remarquable, allant de cours de formation professionnelle à l'information/sensibilisation des résidents producteurs de déchets.

Les associations sectorielles ont collaboré pour transformer les résultats de recherche en guide et cours de formation sur la prévention des accidents du travail. Des fiches techniques, des affiches et des autocollants sur les méthodes de travail sécuritaires et les mesures de prévention (collecte bilatérale, aménagement des camions, exposition aux bioaérosols) ont été distribués aux gestionnaires responsables des collectes de déchets et aux travailleurs.

43 La CSST a joué son rôle de relayeur et d'utilisateur des résultats de recherche en produisant des vidéos sur les risques d'accidents, sur l'aménagement du camion à chargement arrière et sur le travail du chauffeur et de son aide-éboueur.

Enfin, les municipalités ont aussi servi de relayeurs en produisant et distribuant de l'information auprès de leurs résidents - premiers utilisateurs de ce service public - sous forme d'affiches, dépliants et autocollants les sensibilisant aux risques que certains de leurs comportements faisaient courir aux éboueurs (poids excessifs, camouflage de produits dangereux, contenants inappropriés). Certaines municipalités ont également pris soin de prévenir les citoyens des modifications apportées au processus de collecte et de les informer sur les responsabilités à prendre et les gestes, souvent simples, à poser pour protéger la santé et la sécurité des éboueurs. 


\subsection{L'utilisation effective des résultats de recherche : les changements survenus dans le secteur}

Depuis la sortie des deux études, plusieurs changements positifs se sont produits dans le secteur. En voici quelques-uns parmi les plus marquants.

Nous avons demandé aux responsables en santé-sécurité de l'une des neuf entreprises étudiées en 1990-92 de bien vouloir brosser aujourd'hui le portrait des changements survenus chez eux au cours des 10 dernières années. Ils nous ont adressé le bilan suivant, en faisant spontanément référence à divers paliers de recommandations énoncés dans le rapport de recherche de 1992 :

- Chute du taux de fréquence des accidents (avec et sans perte de temps) de $75 \%$ à $34 \%$.

- Baisse du taux de cotisation de l'employeur à la CSST de 11,3\$ à 8,15\$ pour $100 \$$ de masse salariale cotisable.

- Réduction considérable du nombre de blessures au dos et aux épaules autrefois reliées à la levée des charges à bout de bras (camions à chargement latéral = trémie élevée), aux lancers et aux torsions.

- Stabilisation de la main-d'œuvre (baisse du taux de roulement). Formation à l'embauche et formation permanente basées sur la polyvalence des postes et le parrainage par des éboueurs d'expérience. Conséquence: constitution d'un noyau d'éboueurs expérimentés ayant développé une meilleure connaissance des parcours et des risques.

- Information et sensibilisation des résidents ayant mené à la réduction du nombre d'objets et de comportements dangereux (distribution de dépliants, ouverture du centre de tri aux visites d'écoles et de groupes, émissions à la télévision communautaire locale, billets de courtoisie remis aux résidents pris en faute).

Bien entendu, cet exemple de bilan positif ne concerne qu'une entreprise et nous verrons d'ailleurs plus loin que ce même bilan comporte aussi des lacunes, mais il n'en demeure pas moins que la recherche a changé des choses, tant au niveau des entreprises qu'à l'échelle de la province, comme nous allons le voir.

La recherche avait montré que près de $30 \%$ des accidents étaient reliés aux montées et descentes du camion des éboueurs et des chauffeurs à l'aide du marchepied. Une activité de valorisation des résultats de recherche a dès lors été financée sous forme d'expertiseconseil visant à apporter des aménagements ergonomiques aux marchepieds et poignées de préhension de la benne des camions à chargement arrière. Des plans et devis ont été élaborés en conformité avec les normes dans ce domaine et mis à la disposition des clientèles via les associations sectorielles concernées. Tous les camions servant à la collecte des déchets de la ville de Montréal ainsi que ceux de quelques entreprises privées sont maintenant aménagés de façon sécuritaire; cela ne concerne pas seulement les marchepieds mais aussi les dimensions des dégagements, la position et les dimensions des miroirs et rétroviseurs ainsi que l'emplacement des barres de préhension; en fait : tout l'aménagement du poste arrière des véhicules a été revu.

Les efforts excessifs et trop nombreux devraient devenir bientôt, souhaitons-le, chose du passé : des actions ont été entreprises afin d'accélérer la diffusion de la collecte semiautomatisée avec bacs roulants qui ont en outre l'avantage de réduire les contacts des éboueurs avec les objets et produits dangereux. De concert avec les entreprises privées, plusieurs municipalités ont décidé d'appliquer enfin les articles du règlement provincial sur la gestion des déchets solides concernant les poids maximums des contenants. 
Lorsque des contenants et contenus sont hors normes, les éboueurs en avisent dorénavant les citoyens au moyen d'autocollants.

Les associations sectorielles concernées ont collaboré à la rédaction d'une clause type en santé-sécurité devant être insérée dans le cahier des charges des entreprises privées soumissionnaires pour les contrats en sous-traitance. Le cours de formation sur la prévention des accidents du travail conçu par ces mêmes associations sectorielles a été suivi par 630 éboueurs et contremaîtres, et le guide de formation distribué à plus de 650 exemplaires.

51 Les résultats de recherche ayant démontré l'urgence d'agir, la CSST a mis en place un Programme d'Intervention Intégré (PII) sur la prévention des risques biologiques et y a invité les personnes et organismes œuvrant dans le secteur. C'est ainsi que la fiche technique sur la prévention des risques reliés aux bioaérosols a été distribuée aux 2800 éboueurs et chauffeurs de la province. Par ailleurs, dans le but d'améliorer les interventions de ses inspecteurs dans cette industrie peu connue, la CSST a organisé pour eux une journée d'information sous forme d'ateliers sur l'étude des accidents dans la collecte des déchets. Une formation sur les risques spécifiques par métier et une autre sur les dangers de la collecte bilatérale leur ont aussi été dispensées.

Une action concertée des inspecteurs de la CSST avec le milieu de travail pour prohiber la collecte bilatérale et un groupe de travail réunissant les municipalités et les entreprises privées pour la prévention des risques dans la collecte des ordures ménagères (initiative de la CSST et des deux ASP) ont été mis en place.

La Régie régionale de la santé et des services sociaux de Montréal et certains centres locaux de services communautaires (CLSC, Équipes de santé au travail) utilisent les résultats de recherche dans leurs programmes de surveillance des risques dans les entreprises.

Enfin, en ce qui concerne la recherche et le développement, les deux projets initiaux ont suscité des études de deuxième génération, faisant naître des demandes dans les milieux de travail pour que soient réalisées des études "satellites" sur les équipements de protection, les centres de tri et compostage, l'évaluation de la collecte avec bacs roulants et de la collecte entièrement automatisée avec bras articulé. Les connaissances et l'expertise dans ce domaine se sont donc élargies considérablement.

\section{Discussion : les limites au transfert et à l'utilisation des connaissances scientifiques}

Les nombreuses retombées positives observées dans le secteur de la collecte des déchets ne doivent cependant pas laisser croire que toutes les questions de santé et de sécurité de cette industrie ont été résolues, ni que la totalité des recommandations ont été appliquées, loin de là. Il existe des limites aux possibilités de transfert et d'utilisation des connaissances scientifiques. Elles sont de plusieurs ordres.

En premier lieu, il faut admettre que les améliorations en santé et sécurité ne sont pas toujours le fruit de la recherche. C'est ainsi que l'introduction de la collecte semiautomatisée avec bacs roulants, par exemple, est tout autant le résultat des efforts commerciaux pour diffuser la vente de ces bacs à travers le continent que la conséquence 
des recommandations issues de l'étude ; on peut même avancer que l'étude a donné un sérieux coup de main aux vendeurs d'équipements.

Plus inquiétant, certaines améliorations risquent de déplacer les problèmes vers d'autres types de lésions. Les nouvelles technologies, si elles réduisent le nombre d'accidents, semblent provoquer l'apparition de nouveaux types de problèmes, comme les lésions musculo-squelettiques entre autres; c'est ce que démontrent les études satellites de deuxième génération qui ont fait suite à l'étude principale. Dans la compagnie mentionnée précédemment, par exemple, on relève maintenant des problèmes posturaux reliés à la conception de la cabine des camions destinés à la collecte entièrement automatisée (angle de vision de l'écran-caméra, localisation des manettes de commande, position du siège, hauteur du dossier), problèmes qui génèrent des mouvements répétitifs sous postures contraignantes entraînant des tendinites et bursites. On note aussi l'apparition de symptômes liés à l'inactivité (embonpoint, baisse des capacités cardiorespiratoires, entorses lors des démarrages à froid), le refus des travailleurs de porter les chaussures protectrices et surtout la lenteur du processus de sensibilisation des citoyens. En effet, malgré les nouvelles réglementations, on constate encore la présence de matières déposées en vrac à côté des bacs, de contenants non réglementaires et de petits sacs de plastique obligeant souvent l'éboueur à descendre du camion et à exécuter de nombreuses flexions.

Cependant, les résistances les plus fortes à l'utilisation des résultats de recherche sont d'un autre ordre que les aspects simplement techniques. Le transfert des connaissances a peu d'effet quand il s'agit de facteurs sur lesquels on a peu d'emprise, comme les comportements individuels des résidents et des travailleurs, les facteurs personnels, ou certains aspects macroscopiques de l'organisation du travail. Dans la collecte des déchets, par exemple, les possibilités d'application des recommandations varient selon qu'il s'agit du secteur public ou privé : protégés par des conventions collectives solides et par des syndicats fortement revendicatifs, les travailleurs municipaux ont plus de marge de manœuvre que ceux des entreprises privées pour exiger des correctifs à leurs conditions de travail.

Nous avons constaté, finalement, que les résultats de recherche avaient peu de chance d'être appliqués quand ils entraient en conflit avec les intérêts économiques et politiques à court terme. La collecte des déchets est un travail à forfait, majoritairement exécuté pour les municipalités par des entreprises sous-traitantes. La taxe municipale pour la collecte des déchets n'a pas changé depuis 20 ans. Dans certaines municipalités, l'entrepreneur offre même d'effectuer la collecte à un coût moindre qu'il y a 10 ans. Tant que subsistera la règle de l'octroi des contrats au plus bas soumissionnaire et que nous refuserons - en tant que citoyens payeurs de taxes municipales - premièrement de voir ce que coûte vraiment ce service public, deuxièmement d'en payer le prix réel, le risque majeur pour la santé et la sécurité des éboueurs restera le rythme obligatoirement rapide de la collecte, avec son impact sur la charge de travail. Ainsi, beaucoup d'éboueurs continueront à soulever leurs 16 tonnes quotidiennes en courant $11 \mathrm{~km}$ d'un côté à l'autre de la rue (malgré les consignes interdisant la collecte bilatérale), sans protection, aux côtés d'un camion peu adapté aux exigences de la tâche. Pour chaque heure de travail, ils continueront à faire 53 montées-descentes du marchepied, 482 flexions, 203 lancers, 159 torsions. 


\section{Conclusion}

60 relayeurs de premier ordre. travail. sur le terrain.
En dépit des résistances qui viennent d'être évoquées, nombreux sont les signes montrant que la recherche a laissé des traces durables dans le secteur, à commencer par une meilleure connaissance du travail des éboueurs et par la prise de conscience des risques reliés à la charge de travail et à l'organisation humaine et technique de la collecte.

On peut se demander également si cette recherche particulière n'a pas contribué de façon significative au développement même de l'approche institutionnelle en matière de transfert des connaissances, ainsi qu'au raffinement des modèles discutés dans la littérature sur le sujet. Par exemple, on sait maintenant que les relayeurs relaient dans les deux sens, que les choix relatifs au comité de suivi (composition et rôles) sont importants, que l'engagement des chercheurs dans la diffusion des résultats aux milieux de travail et dans le grand public est un élément clé du transfert, que les hygiénistes et les ergonomes - qui occupent une position privilégiée à l'interface entre action et savoir - sont des

Plusieurs éléments, caractéristiques de l'approche développée au fil des ans par l'équipe de recherche, ont contribué à ce que les résultats soient connus et utilisés dans les milieux de travail. Le comité de suivi paritaire (entreprises, syndicats, travailleurs) a piloté la recherche, s'est approprié les résultats et les a diffusés. L'utilisation de méthodologies impliquant la présence des chercheurs sur le terrain pendant des périodes prolongées a fait de ceux-ci des figures familières dans le monde de la collecte des déchets, si bien que le contact entre les chercheurs et les acteurs influents dans le domaine ainsi qu'avec les principales entreprises privées et publiques s'est maintenu longtemps après l'étude initiale. La participation des chercheurs à la conception des outils de transfert et aux rencontres professionnelles dans les milieux concernés a tout naturellement conduit à l'émergence d'autres relayeurs spontanés dans les milieux de

En somme, les effets de la stratégie de transfert des résultats de recherche adoptée pour les éboueurs ont confirmé la pertinence et l'utilité des éléments clés de l'approche. Si l'on veut que les résultats des recherches scientifiques soient transférés dans les milieux de travail, il importe donc de réunir les conditions suivantes : ciblage des « bons » relayeurs, établissement de liens interpersonnels entre ceux-ci et les chercheurs, association des futurs utilisateurs et relayeurs dès la conception des projets, évaluation rigoureuse de la qualité scientifique de la recherche, engagement des chercheurs dans les activités de transfert, diffusion des résultats au moyen d'outils accessibles à une variété de clientèles

Ces principes ne sont toutefois pas rigides. Ainsi, selon le type de recherche, l'objet d'étude, le besoin exprimé, ou le problème abordé, le transfert prendra des formes et des moyens variés. Dans la mesure où elle n'entraîne par de remaniement profond dans la gestion des opérations, la diffusion d'une innovation technologique, par exemple, nécessitera de la part des acteurs un engagement personnel moindre que celui qui serait requis pour un changement organisationnel profond à l'échelle de tout un secteur.

5 En plus d'être flexibles, ces principes sont aussi source de questionnement. Par exemple, si le ciblage des bons relayeurs est une condition indispensable, on sait aussi que les plus influents d'entre eux ont parfois des intérêts divergents. Faut-il continuer à les asseoir 
tous autour de la même table du comité de suivi? Nous sommes d'avis que les chercheurs ont intérêt à tous les inclure dans les discussions, même si parfois cela risque de « déranger » momentanément la recherche. C'est le prix à payer pour obtenir aussi les avantages du paritarisme: accès quasi illimité aux données et aux observations de terrain, et certitude qu'une fois le "bébé » adopté et baptisé, ses parrains vont se l'approprier et le défendre ardemment. De toute façon, il ne faut pas se faire trop d'illusions en ce qui concerne l'objectivité des décideurs : il arrive souvent qu'à la fin d'une étude les recommandations adoptées se limitent au peu qui fait consensus entre les parties et, pour le reste, chacun prend ce qui l'arrange en fonction de ses besoins. S'il est une chose sur laquelle les chercheurs ont peu de contrôle, c'est bien celle-là.

Enfin, pour juger de l'efficience du transfert, il faut prendre du temps et du recul. Dans ce domaine, il peut y avoir de belles surprises. Si l'on avait dû parier au départ sur les chances respectives de transfert de connaissances des études sur les éboueurs et de l'étude sur les horaires de travail dans la pétrochimie (Bourdouxhe et coll., 1997, \#2), peu de personnes auraient risqué leur mise sur les éboueurs. En effet, la disparité des contextes semblait favoriser les opérateurs de la raffinerie, qui travaillent dans une seule grosse entreprise structurée, organisée, syndiquée, performante et disposant de grands moyens, alors que les éboueurs appartiennent à un secteur indiscipliné, composé d'une multitude d'entreprises hétéroclites et où la volonté d'amélioration, au début du moins, était loin d'être évidente, ni partagée. Pourtant, à terme, on est bien forcé de constater que le secteur de la collecte des déchets est celui où les changements ont été les plus nombreux et les plus durables, alors que la raffinerie s'est montrée fermée à tout changement ou suivi scientifique éventuel (l'autopsie de ce demi-échec a été rapportée dans Pistes, vol. 1 (1), 1999). Le paradoxe ne surprend qu'à moitié, cependant, quand on analyse de façon détaillée la présence ou l'absence des "conditions gagnantes » du schéma de transfert dans chacune des deux situations. Malgré son apparente anarchie, par contraste avec la raffinerie, l'industrie de la collecte des déchets s'est révélée suffisamment motivée et organisée pour établir avec les relayeurs et avec les chercheurs des liens de confiance et d'intérêt mutuels menant à une coopération durable. Aujourd'hui encore, les principaux acteurs du secteur sont présents et ils restent motivés, acceptant même pour documenter cet article de poser un regard critique sur leurs propres activités de prévention.

\section{BIBLIOGRAPHIE}

Association of Workers' Compensation Boards of Canada (AWCBC)/ Association des Commissions des accidents du travail du Canada (ACATC) (2001). Public Forum on Knowledge Transfer. Toronto, Canada, November 18-19.

Bourdouxhe, M. (2002). La collecte des déchets domestiques. Dans J.M. Stellman (ed.) Encyclopédie de sécurité et de santé au travail, $3^{\mathrm{e}}$ édition française, BIT-ILO, Genève, ISBN 92-2-209816-1, Volume III : 101.13-101.16.

Perspectives interdisciplinaires sur le travail et la santé, 5-1 | 2003 
Bourdouxhe, M., Toulouse, G., Quéinnec, Y. (1999). Les défis et mirages de la rechercheintervention sur les temps de travail. PISTES, 1, 1. https://pistes.revues.org/3841

Bourdouxhe, M. (1998). Domestic waste collection. I.L.O. Encyclopaedia of Occupational Health and Safety. Geneva : ILO. Fourth Edition. vol. III, 101.12-101.14.

Bourdouxhe, M., Quéinnec, Y., Granger, D., Baril, R., Guertin, S., Massicotte, P., Levy, M., Simard, M., Lemay, F., Casanova, C. (1997). Effets de l'horaire rotatif de 12 heures sur la santé et la sécurité des opérateurs d'une raffinerie de produits pétroliers - Phase I : enquête, diagnostic, pistes de réflexion pour des aménagements. IRSST, Montréal, R-162.

Bourdouxhe, M. (1994). Accident Hazards Associated with Domestic Waste Collection. The Society for the Advancement of Socio-Economics, Sixth International Conference « The Emergent World, Economy and Society - Risk, Public Safety, and the Environment ", Paris, 15-17 juillet.

Bourdouxhe, M., Guertin, S., Cloutier, E. (1992). Étude des risques d'accident dans la collecte des ordures ménagères. IRSST, R-061, Montréal.

Cloutier, E. (1994). The effect of age on safety and work practices among domestic trash collectors in Quebec. Safety Science. 17, 291-308.

Dissanayake, W. (1986). Communication Models and Knowledge Generation, Dissemination and Utilization Activities : a Historical Survey. In G.M. Beal, W. Dissanayake and S. Konoshima (eds) Knowledge Generation, Exchange and Utilization. Westview Press, 293 p.

Fondation canadienne de la recherche sur les services de santé (FCRSS) (1999). Questions de liens et d'échanges entre les chercheurs et les décideurs. Sommaire d'un atelier convoqué par la Fondation, document de référence, mai, $29 \mathrm{p}$.

Fonds de la recherche en santé du Québec (FRSQ) (1997). FRSQ-Actualités, Bulletin d'information, semaine du 27 octobre, p. 1.

Gélinas, A. (1990). Les fondements du transfert des connaissances. In CQRS (ed.) Le transfert des connaissances en recherche sociale, Actes du Forum du conseil québécois de la recherche sociale, Montréal, avril, 17-38.

Goyer, N., Lavoie, J. (2001). Emissions of Chemical Compounds and Bioaerosols During the Secondary Treatment of Paper Mill Effluents. American Industrial Hygiene Association Journal, 62, 3 , 330-341.

Gratton, L., Bourdouxhe, M., Lavoie, J., Bouliane, P., Guertin, S. (2001). Transfer and Use of the Results of Two Research Projects on Domestic Waste Collection, Affiche présentée au Forum Transfert des connaissances / Knowledge Transfer, ACATC/AWCBC, Toronto, Canada, 19 novembre.

Huberman, M., Gather-Thurler, M. (1991). De la recherche à la pratique. éléments de base. Berne : Peter Lang Verlag, 115 p.

Landry, R. (1999). Les liens et les échanges comme déterminants de l'utilisation de la recherche : Analyse raisonnée et quelques faits provenant d'autres pays. Dans Questions de liens et d'échanges entre les chercheurs et les décideurs, document de référence, Fondation canadienne de la recherche sur les services de santé, annexe 1, mai, p. 20-21.

Landry, R., Amara, N., Lamari, M. (2000). Évaluation de l'utilisation de la recherche sociale subventionnée par le CQRS. Chaire FCRSS/CHSRF sur la dissémination et l'utilisation de la recherche, Université Laval, septembre, $321 \mathrm{p}$.

Lavoie, J., Dunkerley, C.J. (2002). Assessing waste collectors' exposure to bioaerosols. Aerobiologia, 18, 4, 1-9. 
Lavoie, J. (2001) Evaluation of health and safety risks in municipal solid waste recycling plants. Journal of the Air and Waste Management Association, 51, 3, 352-360.

Lavoie, J. (2001). Évaluation de l'exposition des éboueurs aux bioaérosols. Actes du $23^{e}$ congrès de l'Association québécoise pour l'hygiène, la santé et la sécurité du travail (AQHSST), 9-11 mai, Hull, Québec, p. 7-12.

Lavoie, J. (2000). Évaluation de l'exposition des éboueurs aux bioaérosols. R-255, Montréal, IRSST, 24 p.

Lomas, J. (1997). Pour améliorer la diffusion et l'utilisation de la recherche dans le secteur de la santé : la fin des dialogues de sourds. Department of Clinical Epidemiology \& Biostatistics, Centre for Health Economics and Policy Analysis, McMaster University, Ontario, août, 40 p.

Roy, M., Guindon, J.-C., Fortier, L. (1995). Transfert de connaissances - revue de littérature et proposition d'un modèle. IRSST, R-099, $53 \mathrm{p}$.

Teiger, C., Montreuil, S., Wendelen, E., Lacomblez, M., Berthelette, D. (1995). L'ergonome, le formateur et le travail. Éducation Permanente, 124, 3, 121.

\section{RÉSUMÉS}

L'étude des mécanismes qui favorisent l'appropriation des résultats de recherche par les milieux de travail révèle l'existence de conditions indispensables à cette transmission. Cet article résume les conditions nécessaires au transfert et présente quelques-unes des principales approches qui sous-tendent les activités de diffusion des résultats de recherche. L'approche choisie est illustrée au moyen de l'exemple de l'étude des risques d'atteinte à la santé et à la sécurité des éboueurs au Québec. Dans ce cas, les conditions favorables étaient réunies : association des futurs utilisateurs dès la conception du protocole de recherche, évaluation rigoureuse de la qualité scientifique de l'étude, ciblage des relayeurs, liens interpersonnels entre ceux-ci et les chercheurs, engagement des chercheurs dans les activités de transfert, diffusion des résultats au moyen d'outils accessibles à la variété des clientèles sur le terrain. Toutefois, il existe des limites technologiques, économiques et politiques - aux possibilités de transfert.

A study of mechanisms supporting the use of research results by workers and managers revealed the existence of certain conditions that are essential to the transmission process. This paper summarizes these conditions and presents a brief review of the principal approaches underlying knowledge transfer activities. The approach selected by the research team is illustrated by means of an example involving the transfer and use of research results relating to the occupational risks of domestic waste collection in Quebec. In this case, the conditions favorable to the transfer were met, namely involvement of future users in the design of the research protocol, rigorous scientific evaluation of the study, targeting of relevant partners, interpersonal contacts between these partners and the researchers, researchers' involvement in transfer activities, and dissemination of results and recommendations using tools accessible to a broad range of users. However, the potential for transfer was limited by certain technological, economic and political considerations.

El estudio de los mecanismos que favorecen la apropiación de los resultados de investigaciones por los ámbitos de trabajo revelan la existencia de condiciones indispensables a esta transmisión. Este artículo resume las condiciones necesarias a la transferencia y presenta algunos de los enfoques principales que sustentan las actividades de difusión de los resultados de la investigación. El enfoque escogido está ilustrado por el ejemplo del estudio de riesgos de perjuicio a la salud y a la seguridad de los basureros en el Quebec. En este caso, se reunían las 
condiciones favorables : asociación de los utilizadores futuros desde la concepción del protocolo de investigación, evaluación rigurosa de la calidad scientífica del estudio, selección de los emisores, vínculos interpersonales entre éstos y los investigadores, compromisión de los investigadores en las actividades de transferencia, difusión de los resultados con instrumentos accesibles a la variedad de las clientelas en el terreno. Sin embargo, existen límites- tecnológicas, económicas y políticas- a las posibilidades de transferencia.

\section{INDEX}

Palabras claves : transferencia de conocimientos, enfoques teóricos, condiciones favorables, estudio monográfico, recogida de residuos

Mots-clés : transfert des connaissances, approches théoriques, conditions favorables, étude de cas, collecte des déchets

Keywords : knowledge transfer, theoretical approaches, favorable conditions, case study, domestic waste collection

\section{AUTEURS}

\section{MADELEINE BOURDOUXHE}

Institut de recherche Robert-Sauvé en santé et en sécurité du travail (IRSST), 505, boul. De Maisonneuve Ouest, Montréal (Québec) Canada - H3A 3C2, bourdouxhe.madeleine@irsst.qc.ca

\section{LAURENT GRATTON}

Institut de recherche Robert-Sauvé en santé et en sécurité du travail (IRSST), 505, boul. De Maisonneuve Ouest, Montréal (Québec) Canada - H3A 3C2, gratton.laurent@irsst.qc.ca 